Journal of Engineering and Applied Sciences 14 (5): 1538-1548, 2019

ISSN: 1816-949X

(C) Medwell Journals, 2019

\title{
New Correlation Equations for Finned Tube Heat Exchangers
}

\author{
Sajida Lafta Ghashim \\ Department of Mechanical Engineering, University of Baghdad, Baghdad, Iraq
}

\begin{abstract}
A new correlation equations were conducted on heat transfer parameters of air-side in circular finned-tube heat exchangers of staggered and in line arrangement. A Nusselt number and Euler number correlation are derived. The effects of fin height, fin spacing, fin pitch, tube outside diameter, fin diameter, transverse tube pitch, longitudinal tube pitch and number of rows are reflected in the correlations. The study covered the range of Reynolds number $\left(8 \times 10^{3}-5 \times 10^{4}\right)$. Number of bundles used in this correlation 56 staggered and 33 in line. A numerical solution based on (DGA V1.00) and Excel programs were used to find constants of correlation equations. Also, Tecplot program is used to draw curves of results. Results show maximum deviation are in Nusselt number and Euler number correlations $(6,18 \%$ for staggered and $10,11 \%$ for in line), respectively in addition, the effect of fin spacing or fin height on Nusselt number and Euler number in one bundle (S1 staggered and I1 in line) are investigated. Results showed that in staggered bundle arrangement S1, the Nu number increased and Eu number decreased when the fin spacing increased and fin height decreased. While the effect of decreased fin spacing and increased fin height led to in increased Nu number and Eu number at bundle I1 in line arrangement.
\end{abstract}

Key words: Circular finned tubes, staggered array, in line bundle arrangement, heat transfer coefficient, fin diameter, transverse tube pitch

\section{INTRODUCTION}

Finned tube one of most imported method to enhance heat transfer in heat exchanger. Literature survey reveals that correlation for a Nusselt and friction factor is derived for both in-line and staggered arrays tubes arranged in heat exchanger. Briggs and Young (1963) derived new correlation equation of Nusselt number. The scope of the research was to study effect three values of the fin thickness $(0.457,1.06$ and 2.02) $\mathrm{mm}$ on heat transfer coefficient. The results showed, heat transfer coefficient decreased when value of fin thickness increased. Haaf (1988) explored a new pressure drop correlation for both in-line and staggered arrays. Mon (2003) investigated numerically pressure drop and heat transfer on finned circular tubes heat exchanger. The study cover the range of Reynolds number from 5000-70000. Continuity, momentum, energy and the $(\mathrm{k}-\varepsilon)$ turbulence model used in the numerical analysis. CFD code Fluent (ANSYS 15) and Gambit 2.2.30 are used to solve mathematical equations and its package based on finite volume method. The result for both in-line and staggered tubes are presented by temperature distribution between the fins, local Nusselt number along tube wall of bundle and temperature countor. Also, new Nusselt number and pressure drop correlation is obtained. Taler (2012) showed numerically the effect of extended surfaces in a cross-flow heat exchangers and new correlation equations obtained.
Nonlinear regression method was used to find constant of correlation. Fajiang et al. (2012) studied experimentally heat transfer for flow spiral finned tube heat exchanger. The experiments were conducted with $\mathrm{Re}$ number $5 \times 10^{3} \sim 5.5 \times 10^{4}$ and using 13 spiral finned tube bundles heat exchangers. Diameter of base tube $(\mathrm{d}=32 \mathrm{~mm})$ range of fin pitch $(0.22 \sim 0.5)$ fin height $0.22 \sim 0.5$, transverse tube pitch 2 3.3125, longitudinal tube pitch 2 3.3125. Nusselt number and Euler number correlations were obtained. Also, the maximum deviation in heat transfer correlations and pressure drop correlations equal to $( \pm 15,15 \%$ in staggered while \pm 25 and $\pm 30 \%$ for the in-line), respectively. Anoop et al. (2015) studied numerically and experimentally flow over finned tube in air heat exchanger. numerical simulations carried out by using CFD Code Fluent 6.3 for three dimensional and steady state. The flow was governed by the Navier-Stokes equations, continuity equation and the RNG $(\mathrm{k}-\varepsilon)$ material of tube and fin is alloy steel. The numerical study has been done with Reynolds number $2000<\operatorname{Re}<50,000$ for laminar and turbulent flow. Experimental study was conducted at 6 different Reynolds numbers between 1000 and 8000 . experiments were conducted in the vertical wind tunnel. The dimension of length of the tube was $400 \mathrm{~mm}$. Wais (2016) investigated numerically effect of 3 types of fin thickness on heat transfer in single row fin tube cross flow heat exchanger. ANSYS CFX program are used also, to perform model study three-dimensional flow and heat 
transfer parameters. The results show when increase fin thickness, the value of heat transfer decreased. The results compared with available correlations to check the numerical calculation.

In the present study, A Nusselt and Euler number correlation equations are derived. Effect of 7 variables in circular finned-tube heat exchangers are reflected in the correlations. Derivation of correlation equations based on dimensions of bundles of finned-tube heat exchangers of staggered and in line arrangement from the previous investigations by Mon (2003). Moreover, effect of fin spacing or fin height on Nusselt number and Euler number in one bundle for staggered and in line bundle arrangement are investigated. The study covered the range of Reynolds number $\left(8 \times 10^{3}-5 \times 10^{4}\right)$.

\section{MATERIALS AND METHODS}

Mathematical modeling: There are seven variables as shown in Fig. 1 and 2 that affect on heat transfer coefficient and friction factor in circular finned-tube heat exchangers of staggered and in line arrangement. The variables:

- Fin height $\left(\mathrm{h}_{\mathrm{f}}\right)$

- Fin pitch $\left(\mathrm{S}_{\mathrm{f}}\right)$

- fin diameter $\left(\mathrm{d}_{\mathrm{f}}\right)$

- $\quad$ Fin Spacing (S)

- Tube outside diameter (d)

- Transverse tube pitch $\left(\mathrm{S}_{\mathrm{t}}\right)$

- Longitudinal tube pitch $\left(\mathrm{S}_{\mathrm{L}}\right)$

- Number of rows $(Z)$

Selection of data: Dimensions of bundles used for staggered and in-line arrangement as shown in Table 1 and 2, all dimensions are in $\mathrm{mm}$. Depth of all bundles were considered with four rows except for the staggered bundle S6 and in-line I4 were performed with 2-6 rows and 3-5 rows, respectively. The equation for Nusselt number and Euler number are:

$$
\mathrm{Nu}=\mathrm{CRe}^{\mathrm{a} 1}\left(\frac{\mathrm{S}_{\mathrm{f}}}{\mathrm{d}}\right)^{\mathrm{a} 2}\left(\frac{\mathrm{S}}{\mathrm{h}_{\mathrm{f}}}\right)^{\mathrm{a} 3}\left(\frac{\mathrm{d}}{\mathrm{S}_{\mathrm{t}}}\right)^{\mathrm{a} 4}\left(\frac{\mathrm{d}}{\mathrm{S}_{\mathrm{L}}}\right)^{\mathrm{a} 5} \mathrm{C}_{\mathrm{Z}}
$$

$$
E u=C e^{a 1}\left(\frac{S_{f}}{d}\right)^{a 2}\left(\frac{s}{h_{f}}\right)^{a 3}\left(\frac{d}{S_{t}}\right)^{a 4}\left(\frac{d}{S_{L}}\right)^{a 5} Z^{a 6}
$$

where $\mathrm{C}$, al -a6 are the unknown constant which are to be determined from the data shown in Table 3 and 4. Several analyses where performed by using (DGA V1.00) and Excel program to find the constants of the Eq. 1 and 2. Also in Eq. 1 and 2 value of $C_{z}$ depends of number of row.

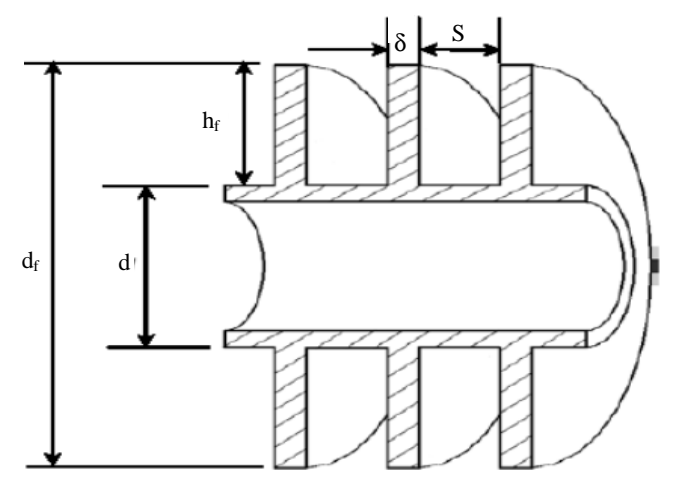

Fig. 1: Circular fin tube section

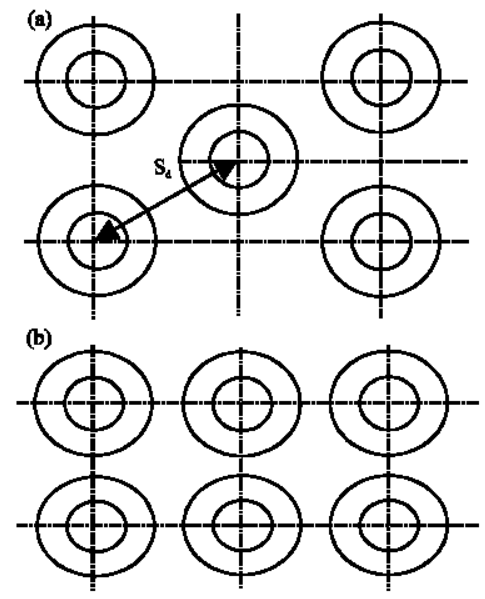

Fig. 2: Arrangements of tubes in bundles; a) Staggered and b) In line

\begin{tabular}{|c|c|c|c|c|c|c|c|c|c|c|c|c|c|c|}
\hline Type & S1 & $\mathrm{S} 2$ & S3 & S4 & S5 & S6 & S7 & S8 & S9 & $\mathrm{S} 10$ & $\mathrm{~S} 11$ & $\mathrm{~S} 12$ & S13 & S14 \\
\hline$\vec{d}$ & 24 & 24 & 24 & 24 & 24 & 24 & 24 & 24 & 24 & 24 & 13.59 & 28 & 24 & 24 \\
\hline$d_{f}$ & 30 & 34 & 38 & 44 & 48 & 34 & 34 & 44 & 34 & 34 & 23.59 & 38 & 34 & 34 \\
\hline$h_{f}$ & 3 & 5 & 7 & 10 & 12 & 5 & 5 & 10 & 5 & 5 & 5 & 5 & 5 & 5 \\
\hline$\delta$ & 0.5 & 0.5 & 0.5 & 0.5 & 0.5 & 0.5 & 0.5 & 0.5 & 0.3 & 0.6 & 0.5 & 0.5 & 0.5 & 0.5 \\
\hline$S$ & 2 & 2 & 2 & 2 & 2 & 1.6 & 4 & 0.7 & 2 & 2 & 2 & 2 & 2 & 2 \\
\hline $\mathrm{S}_{\mathrm{f}}$ & 2.5 & 2.5 & 2.5 & 2.5 & 2.5 & 2.1 & 4.5 & 1.2 & 2.3 & 2.6 & 2.5 & 2.5 & 2.5 & 2.5 \\
\hline St & 36 & 40.8 & 45.6 & 52.8 & 57.6 & 40.8 & 40.8 & 52.8 & 40.8 & 40.8 & 28.30 & 45.6 & 48.4 & 64.8 \\
\hline $\mathrm{S}_{\mathrm{L}}$ & 31.17 & 35.33 & 39.49 & 45.73 & 49.88 & 35.33 & 35.33 & 45.73 & 35.33 & 35.33 & 24.52 & 39.49 & 41.92 & 56.12 \\
\hline Z & 4 & 4 & 4 & 4 & 4 & $2-6$ & 4 & 4 & 4 & 4 & 4 & 4 & 4 & 4 \\
\hline
\end{tabular}

Table 1: Dimensions of bundles for staggered arrangement (Mon, 2003) 
Table 2: Dimensions of bundles for in line arrangement (Mon, 2003)

\begin{tabular}{llllllllll}
\hline Type & I1 & I2 & I3 & I4 & I5 & I6 & I7 & I8 & I9 \\
\hline $\mathrm{d}$ & 24 & 24 & 24 & 24 & 24 & 13.59 & 24 & 24 & 24 \\
$\mathrm{~d}_{\mathrm{f}}$ & 34 & 38 & 44 & 34 & 34 & 23.59 & 34 & 34 & 34 \\
$\mathrm{~h}_{\mathrm{f}}$ & 5 & 7 & 10 & 5 & 5 & 5 & 5 & 5 & 5 \\
$\delta$ & 0.5 & 0.5 & 0.5 & 0.5 & 0.5 & 0.5 & 0.5 & 0.5 & 0.5 \\
$\mathrm{~S}$ & 2 & 2 & 2 & 1.6 & 4 & 2 & 2 & 2 & 2 \\
$\mathrm{~S}_{\mathrm{f}}$ & 2.5 & 2.5 & 2.5 & 2.1 & 4.5 & 2.5 & 2.5 & 2.5 & 2.5 \\
$\mathrm{~S}_{\mathrm{t}}$ & 40.8 & 45.6 & 52.8 & 40.8 & 40.8 & 28.31 & 47.6 & 40.8 & 408 \\
$\mathrm{~S}_{\mathrm{L}}$ & 40.8 & 45.6 & 52.8 & 40.8 & 40.8 & 28.30 & 47.6 & 47.6 & 478 \\
$\mathrm{Z}$ & 4 & 4 & 4 & $3-5$ & 4 & 4 & 4 & 4 & 4 \\
\hline
\end{tabular}

Table 3: Dimensions of 56 types of staggered arrangement finned tubes in $\mathrm{Nu}$ equation

\begin{tabular}{|c|c|c|c|c|c|c|c|}
\hline & & Ke & $\mathrm{S}_{\mathrm{f}} / \mathrm{d}$ & $S / h_{f}$ & $\mathrm{~d} / \mathrm{S}_{\mathrm{t}}$ & $\mathrm{d} / \mathrm{S}_{\mathrm{L}}$ & $\mathrm{C}_{\mathrm{Z}}$ \\
\hline S6 & 2 & 500 & 0.087500 & 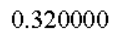 & 5 & 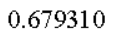 & 0.8656483 \\
\hline & 2 & & & & & & \\
\hline & 2 & & & & & & \\
\hline 6 & 3 & a & & & & & \\
\hline 6 & 3 & 000 & & & & & \\
\hline 6 & 3 & & & & & & \\
\hline & 4 & & & & & & \\
\hline 13 & 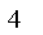 & & & & & & \\
\hline & 4 & & & & & & \\
\hline & 4 & & & & & & \\
\hline & 4 & 500 & & & & & \\
\hline & 4 & & & & & & \\
\hline & 4 & & & & & & \\
\hline & 4 & & & & & & \\
\hline & 4 & & & & & & \\
\hline & 4 & & & & & & \\
\hline & 4 & & & & & & \\
\hline & 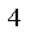 & & & & & & \\
\hline & 4 & & & & & & \\
\hline & 4 & & & & & & \\
\hline & 4 & & & & & & \\
\hline & 4 & & & & & & \\
\hline & 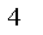 & & & & & & \\
\hline & 4 & & & & & & \\
\hline & 4 & & & & & & \\
\hline & 4 & & & & & & \\
\hline & 4 & & & & & & \\
\hline & 4 & & & & & & \\
\hline & 4 & & & & & & \\
\hline & 4 & & & & & & \\
\hline & 4 & & & & & & \\
\hline & & & & & & & \\
\hline & 4 & & & & & & \\
\hline & 4 & & & & & & \\
\hline & 4 & & & & & & \\
\hline & & & & & & & \\
\hline & 4 & & & & & & \\
\hline & 4 & & & & & & \\
\hline S3 & 4 & & & & & & \\
\hline C4 & 4 & & & & & & \\
\hline & & & & & & & \\
\hline & 4 & & & & & & \\
\hline & 4 & & & & & & \\
\hline 50 & 4 & 430 & & & & & \\
\hline 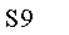 & 4 & & & & & & \\
\hline & & & & & & & \\
\hline & & & & & & & \\
\hline & & & & & & & \\
\hline DI. & 4 & 7. & & & & & \\
\hline & & & & & & & \\
\hline & & & & & & & \\
\hline & & & & & & & \\
\hline S7 & & & & & & & 0046 \\
\hline 50 & & 8000 & $0.00 / 300$ & 0.520000 & 0.500233 & $0.6 / 9510$ & $0.968 / 02$ \\
\hline
\end{tabular}

Table 3: Continue

\begin{tabular}{llllllll}
\hline \multicolumn{1}{l}{ Type } & $\mathrm{Z}$ & $\mathrm{Re}$ & $\mathrm{S} / \mathrm{d}$ & $\mathrm{S} / \mathrm{h}_{\mathrm{f}}$ & $\mathrm{d} / \mathrm{S}_{\mathrm{f}}$ & $\mathrm{d} / \mathrm{S}_{\mathrm{L}}$ & $\mathrm{C}_{\mathrm{Z}}$ \\
\hline $\mathrm{S} 6$ & 5 & 17000 & 0.087500 & 0.320000 & 0.588235 & 0.679310 & 0.9687050 \\
$\mathrm{~S} 6$ & 5 & 43000 & 0.087500 & 0.320000 & 0.588235 & 0.679310 & 0.9687050 \\
$\mathrm{~S} 6$ & 6 & 8600 & 0.087500 & 0.320000 & 0.588235 & 0.679310 & 0.9825061 \\
$\mathrm{~S} 6$ & 6 & 17000 & 0.087500 & 0.320000 & 0.588235 & 0.679310 & 0.9825061 \\
$\mathrm{~S} 6$ & 6 & 43000 & 0.087500 & 0.320000 & 0.588235 & 0.679310 & 0.9825061 \\
$\mathrm{~S} 6$ & 2 & 8600 & 0.087500 & 0.320000 & 0.588235 & 0.679310 & 0.8656483 \\
$\mathrm{~S} 6$ & 2 & 17000 & 0.087500 & 0.320000 & 0.588235 & 0.679310 & 0.8656483 \\
$\mathrm{~S} 6$ & 2 & 43000 & 0.087500 & 0.320000 & 0.588235 & 0.679310 & 0.8656483 \\
$\mathrm{~S} 6$ & 3 & 8600 & 0.087500 & 0.320000 & 0.588235 & 0.679310 & 0.9131156 \\
$\mathrm{~S} 6$ & 3 & 17000 & 0.087500 & 0.320000 & 0.588235 & 0.679310 & 0.9131156 \\
\hline
\end{tabular}

Table 4: Dimensions of 33 types of inline arrangement finned tubes in $\mathrm{Nu}$ equation

\begin{tabular}{llllllll}
\hline Type & $\mathrm{Z}$ & $\mathrm{Re}$ & $\mathrm{S} / \mathrm{d}$ & $\mathrm{S} / \mathrm{h}_{\mathrm{f}}$ & $\mathrm{d} / \mathrm{S}_{\mathrm{t}}$ & $\mathrm{d} / \mathrm{S}_{\mathrm{L}}$ & $\mathrm{C}_{Z}$ \\
\hline $\mathrm{I} 4$ & 3 & 86000 & 0.0875 & 0.320 & 0.5880 & 0.5880 & 1.1 \\
$\mathrm{I} 4$ & 3 & 17000 & 0.0875 & 0.320 & 0.5880 & 0.5880 & 1.1 \\
$\mathrm{I} 4$ & 3 & 43000 & 0.0875 & 0.320 & 0.5880 & 0.5880 & 1.1 \\
$\mathrm{I} 1$ & 4 & 86000 & 0.1040 & 0.500 & 0.5880 & 0.5880 & 1 \\
$\mathrm{I} 2$ & 4 & 86000 & 0.1040 & 0.357 & 0.5260 & 0.5260 & 1 \\
$\mathrm{I} 3$ & 4 & 86000 & 0.1040 & 0.250 & 0.4545 & 0.4545 & 1 \\
$\mathrm{I} 4$ & 4 & 86000 & 0.0875 & 0.320 & 0.5880 & 0.5880 & 1 \\
$\mathrm{I} 5$ & 4 & 86000 & 0.1870 & 0.800 & 0.5880 & 0.5880 & 1 \\
$\mathrm{I} 6$ & 4 & 86000 & 0.1830 & 0.400 & 0.4800 & 0.4800 & 1 \\
I7 & 4 & 86000 & 0.0890 & 0.400 & 0.6140 & 0.6140 & 1 \\
I8 & 4 & 86000 & 0.1040 & 0.400 & 0.5040 & 0.5040 & 1 \\
I9 & 4 & 86000 & 0.1040 & 0.400 & 0.5880 & 0.5040 & 1 \\
I1 & 4 & 17000 & 0.1040 & 0.500 & 0.5880 & 0.5880 & 1 \\
I2 & 4 & 17000 & 0.1040 & 0.357 & 0.5260 & 0.5260 & 1 \\
I3 & 4 & 17000 & 0.1040 & 0.250 & 0.4545 & 0.4545 & 1 \\
I4 & 4 & 17000 & 0.0875 & 0.320 & 0.5880 & 0.5880 & 1 \\
I5 & 4 & 17000 & 0.1870 & 0.800 & 0.5880 & 0.5880 & 1 \\
I6 & 4 & 17000 & 0.1830 & 0.400 & 0.4800 & 0.4800 & 1 \\
I7 & 4 & 17000 & 0.0890 & 0.400 & 0.6140 & 0.6140 & 1 \\
I8 & 4 & 17000 & 0.1040 & 0.400 & 0.5040 & 0.5040 & 1 \\
I9 & 4 & 17000 & 0.1040 & 0.500 & 0.5880 & 0.5880 & 1 \\
I1 & 4 & 43000 & 0.1040 & 0.500 & 0.5880 & 0.5880 & 1 \\
I2 & 4 & 43000 & 0.1040 & 0.357 & 0.5260 & 0.5260 & 1 \\
I3 & 4 & 43000 & 0.1040 & 0.250 & 0.4545 & 0.4545 & 1 \\
I4 & 4 & 43000 & 0.0875 & 0.320 & 0.5880 & 0.5880 & 1 \\
I5 & 4 & 43000 & 0.1870 & 0.800 & 0.5880 & 0.5880 & 1 \\
I6 & 4 & 43000 & 0.1830 & 0.400 & 0.4800 & 0.4800 & 1 \\
I7 & 4 & 43000 & 0.0890 & 0.400 & 0.6140 & 0.6140 & 1 \\
I8 & 4 & 43000 & 0.1040 & 0.400 & 0.5040 & 0.5040 & 1 \\
I9 & 4 & 43000 & 0.1040 & 0.500 & 0.5880 & 0.5880 & 1 \\
I4 & 5 & 86000 & 0.0875 & 0.320 & 0.5880 & 0.5880 & 1 \\
I4 & 5 & 17000 & 0.0875 & 0.320 & 0.5880 & 0.5880 & 1 \\
I4 & 5 & 43000 & 0.0875 & 0.320 & 0.5880 & 0.5880 & 1 \\
\hline & & & & & & &
\end{tabular}

For staggered arrangement:

$$
\begin{aligned}
& C_{Z}=0.71476+0.097819 Z-0.012509 Z^{2}+ \\
& 0.0068758 Z^{3}-0.00013396 Z^{4}
\end{aligned}
$$

For in line arrangement:

$$
C_{Z}=2-0.45 Z+0.05 Z^{2}
$$

The resulting correlation of Nusselt number and Euler number for suitable range of Reynolds number $\left(8 \times 10^{3}-5 \times 10^{4}\right)$ with $Z \geq 2$ has the following form: 


\section{Staggered array:}

$$
\begin{aligned}
& \mathrm{Nu}=0.296 \mathrm{Re}^{0.555}\left(\frac{\mathrm{S}_{\mathrm{f}}}{\mathrm{d}}\right)^{-0.06}\left(\frac{\mathrm{S}}{\mathrm{h}_{\mathrm{f}}}\right)^{0.01}\left(\frac{\mathrm{d}}{\mathrm{S}_{\mathrm{t}}}\right)^{-2.61}\left(\frac{\mathrm{d}}{\mathrm{S}_{\mathrm{L}}}\right)^{2.94} \mathrm{C}_{\mathrm{Z}} \\
& \mathrm{Eu}=37.480 \mathrm{Re}^{-0.23}\left(\frac{\mathrm{S}_{\mathrm{f}}}{\mathrm{d}}\right)^{-0.06}\left(\frac{\mathrm{S}}{\mathrm{h}_{\mathrm{f}}}\right)^{-0.28}\left(\frac{\mathrm{d}}{\mathrm{S}_{\mathrm{t}}}\right)^{16}\left(\frac{\mathrm{d}}{\mathrm{S}_{\mathrm{L}}}\right)^{-15.4} \mathrm{Z}^{0.87}
\end{aligned}
$$

In line array:

$$
\begin{aligned}
& \mathrm{Nu}=0.2127 \mathrm{Re}^{0.661}\left(\frac{\mathrm{S}_{\mathrm{f}}}{\mathrm{d}}\right)^{0.39}\left(\frac{\mathrm{S}}{\mathrm{h}_{\mathrm{f}}}\right)^{-0.031}\left(\frac{\mathrm{d}}{\mathrm{S}_{\mathrm{t}}}\right)^{0.87}\left(\frac{\mathrm{d}}{\mathrm{S}_{\mathrm{L}}}\right)^{-0.61} \mathrm{C}_{\mathrm{Z}} \\
& \mathrm{Eu}=3.889 \mathrm{Re}^{-0.16}\left(\frac{\mathrm{S}_{\mathrm{f}}}{\mathrm{d}}\right)^{0.24}\left(\frac{\mathrm{S}}{\mathrm{h}_{\mathrm{f}}}\right)^{-0.48}\left(\frac{\mathrm{d}}{\mathrm{S}_{\mathrm{t}}}\right)^{2.2}\left(\frac{\mathrm{d}}{\mathrm{S}_{\mathrm{L}}}\right)^{-1.1} \mathrm{Z}^{0.56}
\end{aligned}
$$

\section{Correlations from literature review \\ Heat transfer correlations \\ Mon (2003): For staggered:}

$$
\mathrm{Nu}=0.284 \mathrm{Re}^{0.6} \operatorname{Pr}^{0.333}\left(\frac{\mathrm{A}}{\mathrm{A}_{\mathrm{t}}}\right)^{-0.15} \mathrm{~F}^{-0.075}\left(\frac{\mathrm{S}_{\mathrm{t}}}{\mathrm{S}_{\mathrm{d}}}\right)^{1.06}
$$

For in line:

$$
\mathrm{Eu}=0.356 \operatorname{Re}^{0.6} \operatorname{Pr}^{0.333}\left(\frac{\mathrm{A}}{\mathrm{A}_{\mathrm{t}}}\right)^{-0.15} \mathrm{~F}^{-0.173}\left(\frac{\mathrm{S}_{\mathrm{t}}}{\mathrm{S}_{\mathrm{L}}}\right)^{-0.475}
$$

Briggs and Young (1963): The correlation can be applied for staggered tube arrangement, for Reynolds number range: $1.1 \times 103 \leq \mathrm{Re} \leq 1.8 \times 104,13.49 \mathrm{~mm} \leq \mathrm{d} \leq 40.89 \mathrm{~mm}$, $4.3 \mathrm{~mm} \leq \mathrm{h}_{\mathrm{f}} \leq 16.58 \mathrm{~mm}, 1.82 \mathrm{~mm} \leq \mathrm{S} \leq 2.76 \mathrm{~mm}, 0.33 \mathrm{~mm}$ $\leq \delta \leq 2.02 \mathrm{~mm}, 27.43 \mathrm{~mm} \leq \mathrm{S}_{\mathrm{t}} \leq 110 \mathrm{~mm}, 23.76 \mathrm{~mm} \leq \mathrm{S}_{\mathrm{L}}$ $\leq 96.13 \mathrm{~mm}, Z \geq 4$ :

$$
\mathrm{Nu}=0.134 \operatorname{Re}^{0.681} \operatorname{Pr}^{0.333}\left(\frac{\mathrm{S}}{\mathrm{h}_{\mathrm{f}}}\right)^{0.2}\left(\frac{\mathrm{S}}{\delta}\right)^{0.1134}
$$

Schmidt (1963): The correlation used for Reynolds number range: $1 \times 10^{3} \leq \mathrm{Re} \leq 4 \times 10^{4}, 5 \leq \mathrm{A} / \mathrm{At} \leq 12, \mathrm{Z} \geq$ 3. For staggered: $\mathrm{C}=0.45$, For in line: $\mathrm{C}=0.3$ :

$$
\mathrm{Nu}=\mathrm{CRe}^{0.625} \operatorname{Pr}^{0.333}\left(\frac{\mathrm{A}}{\mathrm{A}_{\mathrm{t}}}\right)^{-0.375}
$$

VDI-GVC (1997): The correlation can be applied for staggered and inline tube arrangement for: $1 \times 10^{3} \leq \mathrm{Re} \leq$ $1 \times 10^{5}, 5 \leq \mathrm{A} / \mathrm{At} \leq 30, \mathrm{Z} \geq 4$. For staggered: $\mathrm{C}=0.38$, for in line: $\mathrm{C}=0.22$ :

$$
\mathrm{Nu}=\mathrm{CRe}^{0.6} \operatorname{Pr}^{0.333}\left(\frac{\mathrm{A}}{\mathrm{A}_{\mathrm{t}}}\right)^{-0.15}
$$

\section{Pressure drop correlations}

Mon (2003): For staggered:

For in line:

$$
\mathrm{Eu}=0.75 \operatorname{Re}^{-0.24} \mathrm{~F}^{-0.49}\left(\frac{\mathrm{S}_{\mathrm{t}}}{\mathrm{S}_{\mathrm{d}}}\right)^{0.64} \mathrm{Z}
$$

$$
\mathrm{Eu}=0.536 \mathrm{Re}^{-0.23}\left(\frac{\mathrm{A}}{\mathrm{A}_{\mathrm{t}}}\right)^{0.068} \mathrm{~F}^{-0.49}\left(\frac{\mathrm{S}_{\mathrm{t}}}{\mathrm{S}_{\mathrm{d}}}\right)^{-2.18} \mathrm{Z}
$$

Haaf (1988): The correlation can be applied for Reynolds number range: $2 \times 10^{2}<\operatorname{Re}<1 \times 10^{4}, Z \geq 4$, for staggered: $\mathrm{C}=$ 4.25 , for in line: $\mathrm{C}=2.5$ :

$$
\mathrm{Eu}=\mathrm{CRe}^{-0.25}\left(\frac{\mathrm{S}_{\mathrm{L}}}{\mathrm{d}}\right)^{0.4} \mathrm{Z}
$$

Robinson and Briggs (1966): The correlation can be applied for Reynolds number range: $2 \times 10^{2}<\mathrm{Re}<1 \times 10^{4}$, for staggered: $\mathrm{C}=4.25$ for in line: $\mathrm{C}=2.5$ :

$$
\mathrm{Eu}=18.93 \mathrm{Re}^{-0.316}\left(\frac{\mathrm{S}_{\mathrm{L}}}{\mathrm{d}}\right)^{-0.92}\left(\frac{\mathrm{S}_{\mathrm{t}}}{\mathrm{S}_{\mathrm{d}}}\right)^{0.515} \mathrm{Z}
$$

\section{RESULTS AND DISCUSSION}

The aim of the research is to attain new correlations. The Nusselt number and Euler number data are correlated for number of row from Eq. 2-6 and Eq. 3-5 for staggered and in line tube banks, respectively. In this new correlations take the effect of seven geometric of heat exchanger in the correlations.

Figure 3 show the Nusselt and Euler number results are plotted versus the $\mathrm{Re}$ number for all bundles staggered arrangement. The $\mathrm{Nu}$ number increase with increases $\operatorname{Re}$ number at different types of bundles. The results illustrated decreases fin height, fin diameter, transverse tube pitch and longitudinal tube pitch causes increase Nusselt number. It was noticed, also that increase fin spacing causes increase Nusselt number. But Euler number affected by same geometric mentioned previously reverse effect.

Figure 4 show the $\mathrm{Nu}$ and Eu number results are plotted versus the $\mathrm{Re}$ number for all bundles in line arrangement. It was concluded from the results that $\mathrm{Nu}$ number increase with increases Re number at different types of bundles and slightly different between the bundles. Moerever, decrease fin height, transverse tube pitch and longitudinal tube pitch causes increase Nusselt number and decrease Euler number. 

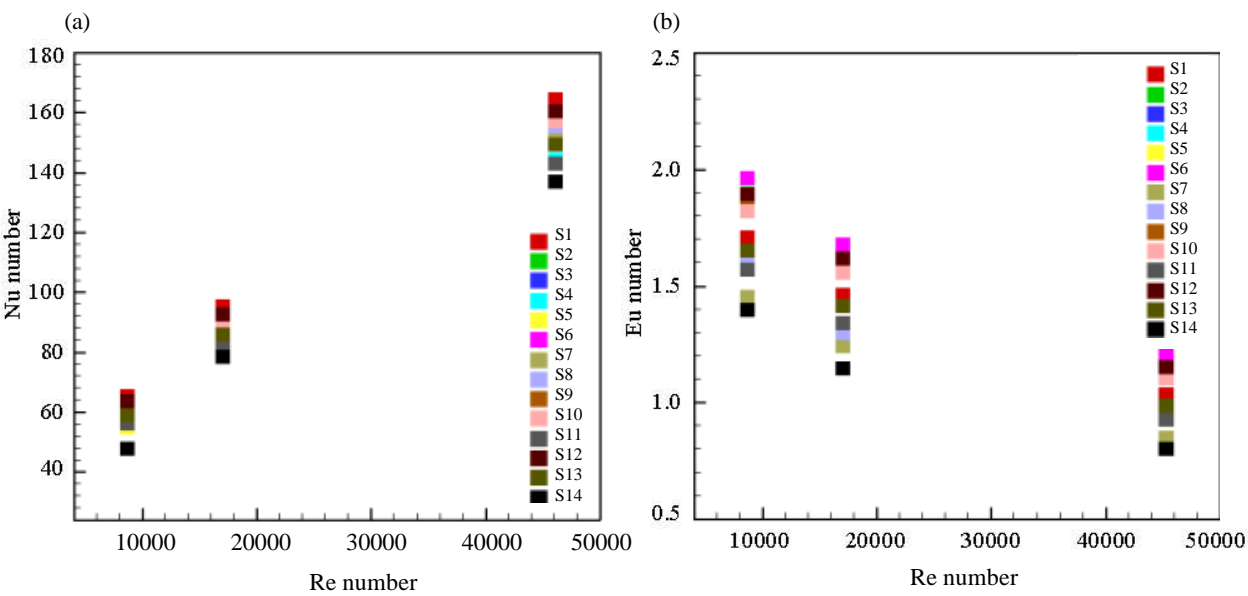

Fig. 3: Nu and Eu number variation with Re number for different staggered bundle arrangement; a) Nu number and b) Eu number
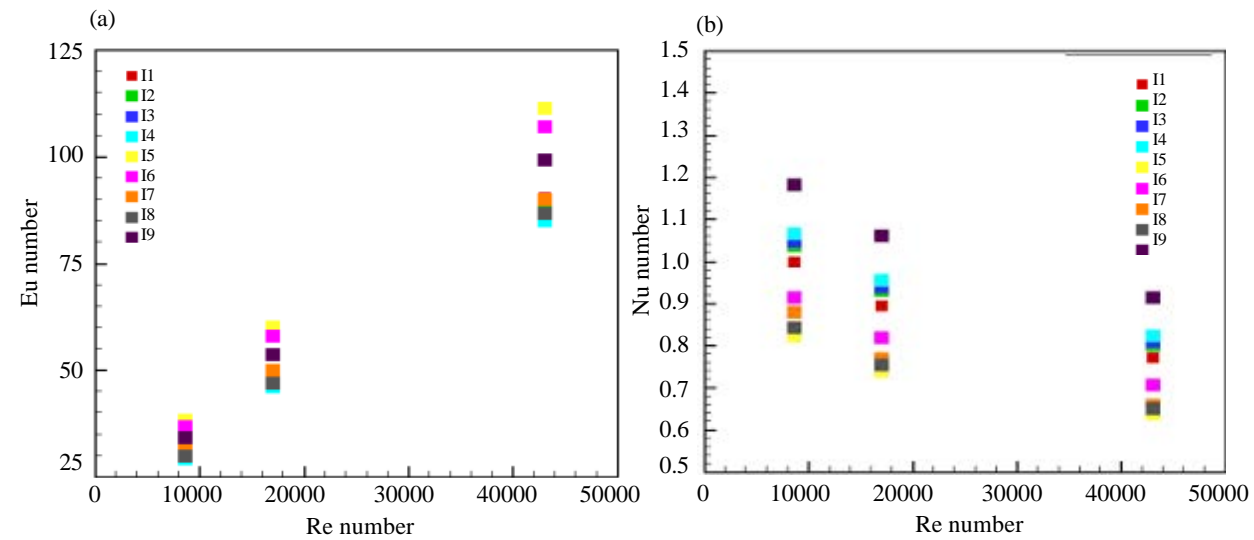

Fig. 4: Nu and Eu number variation with Re number for different inline bundle arrangement; a) Nu number and b) Eu number
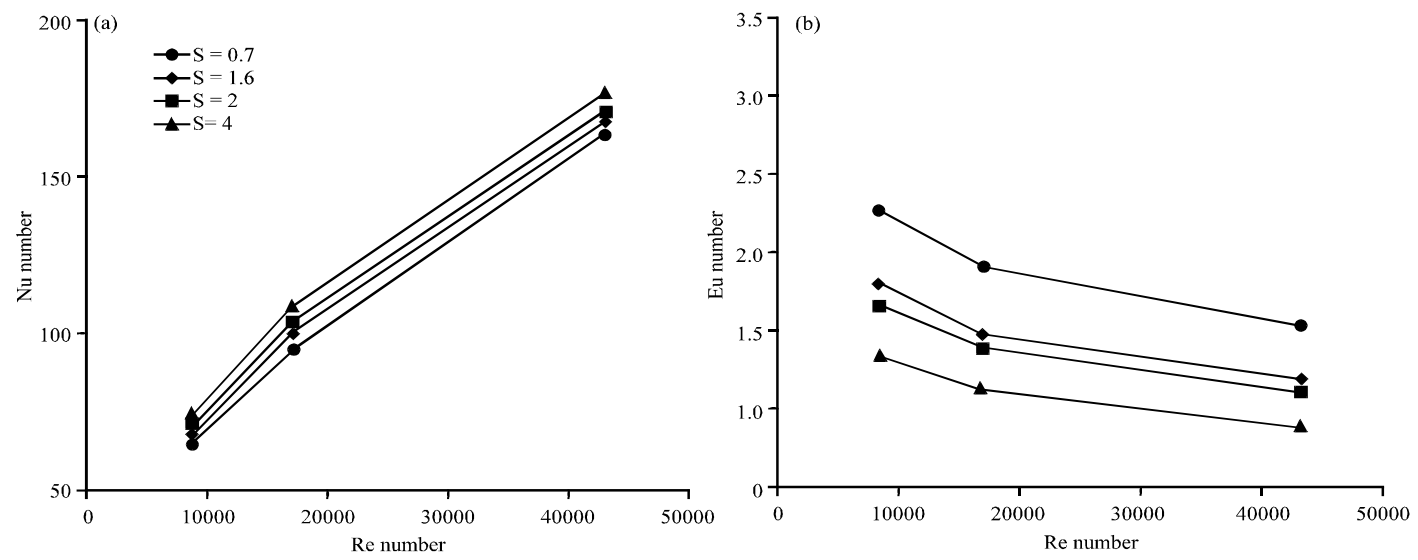

Fig. 5: Nu and Eu number variation with Re number for S1 bundle staggered arrangement at different fin spacing; a) Nu number and b) Eu number

In Fig. 5 and 6 discussed the effect of fin height and fin spacing on $\mathrm{Nu}$ number and Eu number in staggered bundle arrangement $\mathrm{S} 1$ at constant (fin thickness $=0.5$, tube outside diameter $=24$, fin diameter $=30$, transverse 

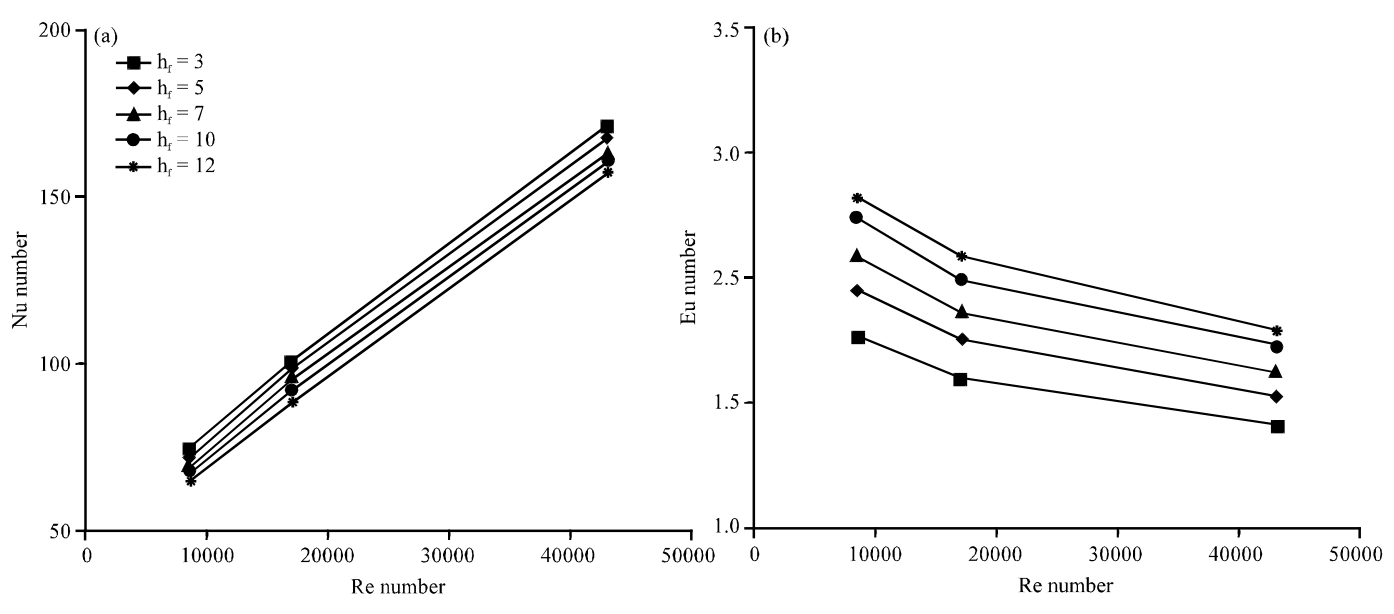

Fig. 6: Nu number variation with Re number for $\mathrm{S} 1$ bundle staggered arrangement at different fin height; a) Nu number and b) Eu number
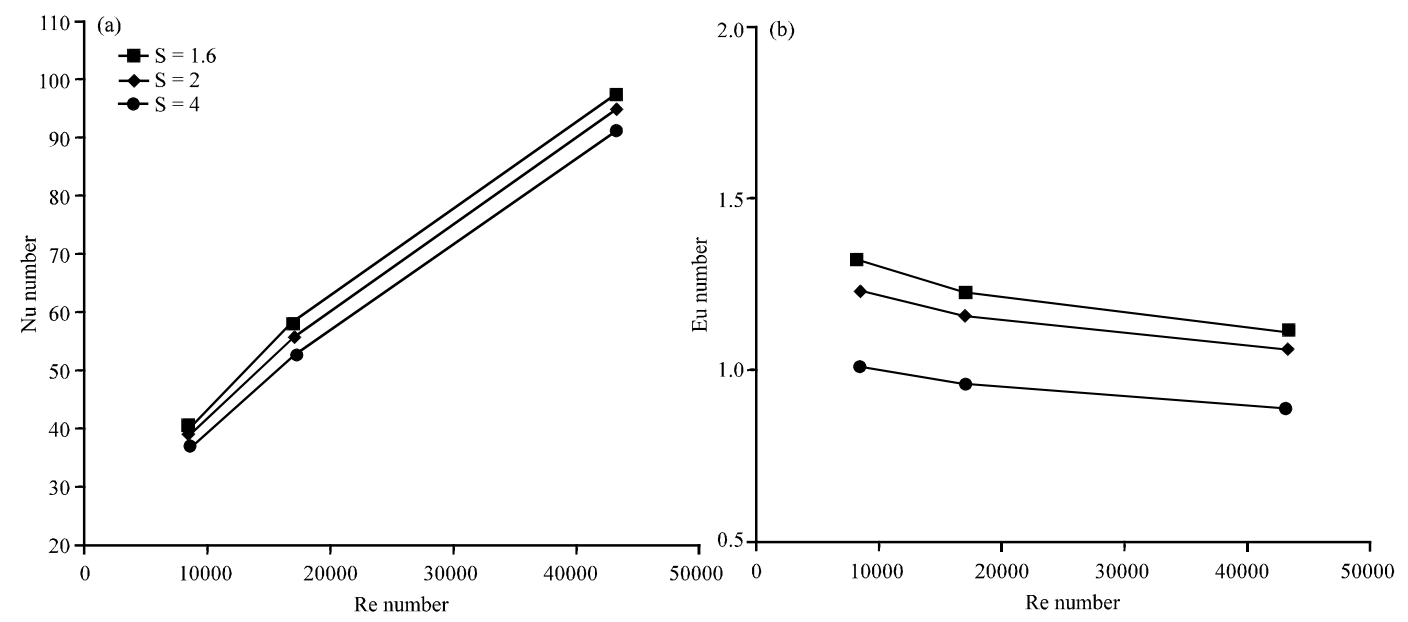

Fig. 7: $\mathrm{Nu}$ and Eu number variation with Re number for I1 bundle in line arrangement at different fin spacing; a) $\mathrm{Nu}$ number and b) Eu number

tube pitch $=$ longitudinal tube pitch $=$ and number of rows $=4$ ). The results illustrated Nu number increased and Eu number decreased when the fin spacing increases from $0.7-4$ and fin height decreased from 12-3.

Figure 7 and 8 show the $\mathrm{Nu}$ number and $\mathrm{Eu}$ number results are plotted versus the Re number for I1 bundle in line arrangement at constant (fin thickness $=0.5$, tube outside diameter $=24$, fin diameter $=34$, transverse tube pitch $=40.8$, longitudinal tube pitch $=40.8$ and number of rows $=4$ ). The results showed that the effect of decreased fin spacing and increased fin height led to in increased $\mathrm{Nu}$ number. Also, similar behavior was noticed for Eu number. From Fig. 9 and 10 , it is evident that the proposed correlations are in good agreement with the equations of Mon (2003).
Comparison with other correlations: To check the result of new correlations, its comparison with the correlations from the literature are shown in Table 5-8, for staggered and in line arrangements tubes. Also, deviation of present correlation was calculated by using the formula:

$$
\text { Deviation }=\frac{\mathrm{Nu}_{\mathrm{Eq}}-\mathrm{Nu}_{\text {Fluent }}}{\mathrm{Nu}_{\mathrm{Eq}}} * 100 \%
$$

The maximum deviation of Nusselt number and Euler number correlation are shown in Table 9-12 $(6,18 \%$ for staggered and $10,11 \%$ for in line), respectively. 

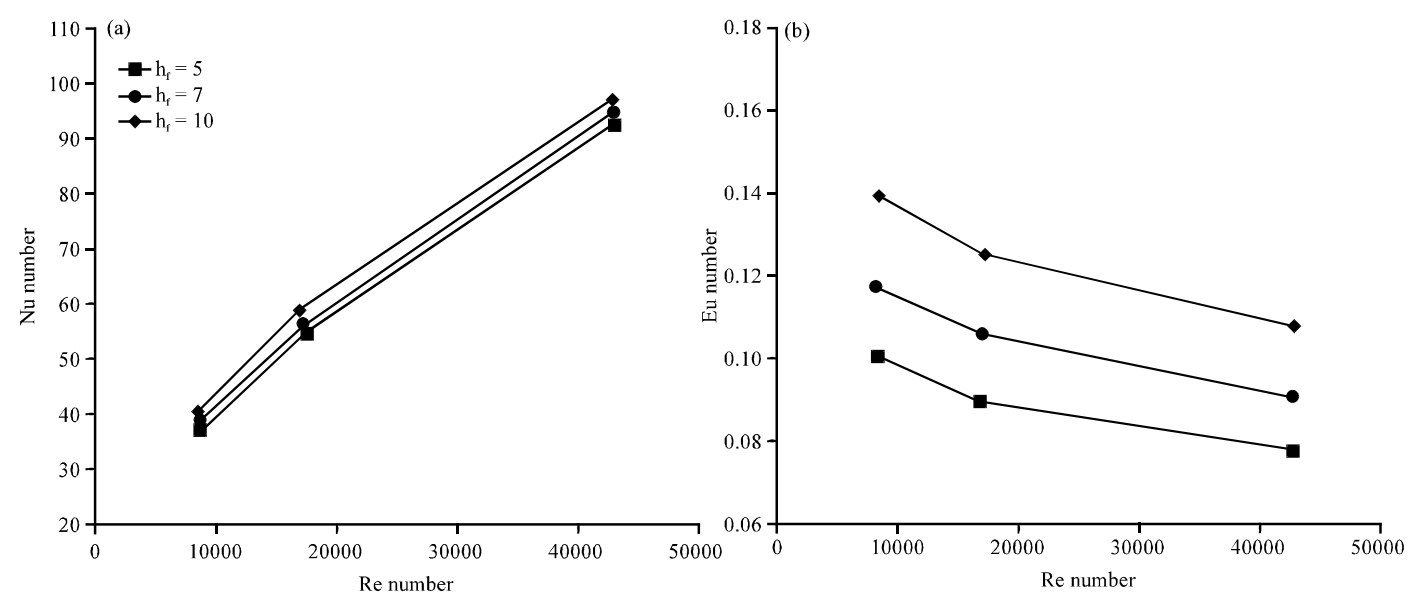

Fig. 8: Nu and Eu number variation with Re number for I1 bundle in line arrangement at different fin height; a) Nu number and b) Eu number
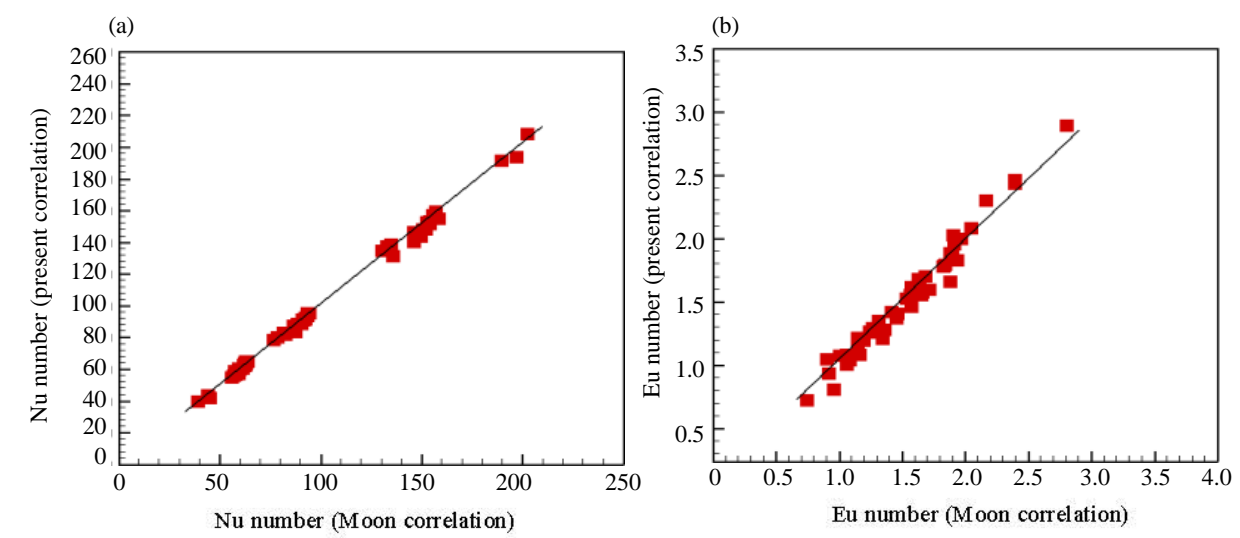

Fig. 9: Comparison of $\mathrm{Nu}$ and Eu number numerical values for staggered finned tube heat exchangers with correlated by Eq 5 and 6; a) Nu number and b) Eu number
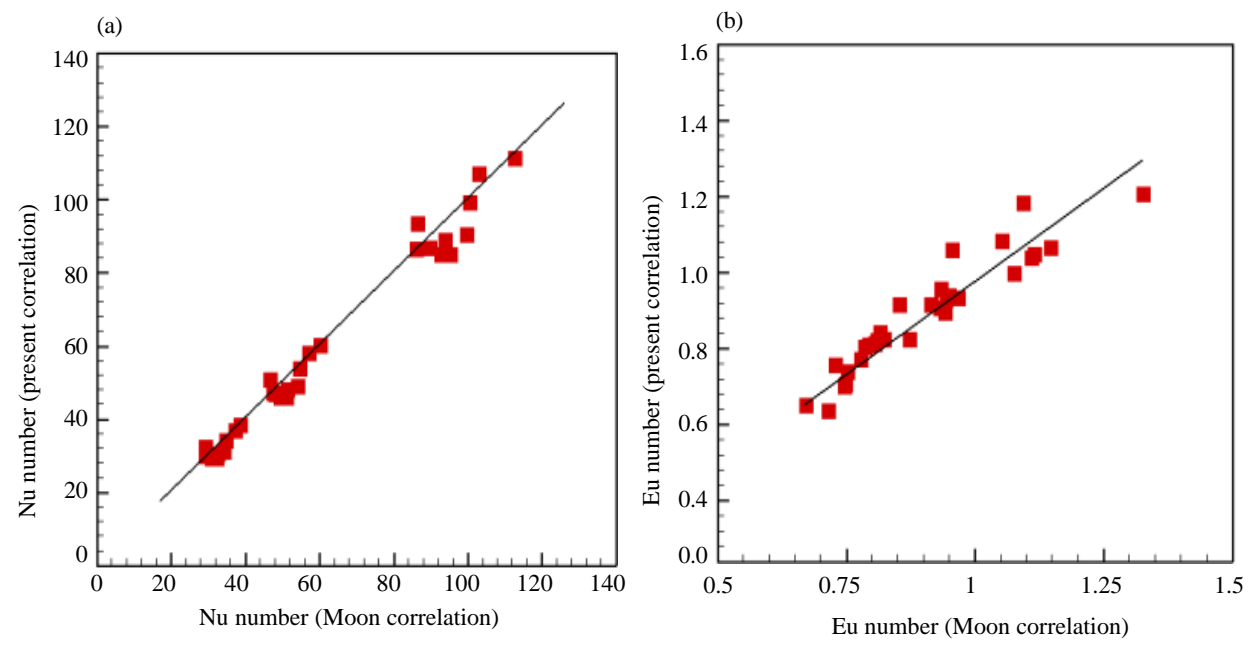

Fig. 10: Comparison of Nu and Eu number numerical values for in line finned tube heat exchangers with correlated by Eq. 7 and 8; a) Nu number and b) Eu number 
J. Eng. Applied Sci., 14 (5): 1538-1548, 2019

Table 5: Comparison Nu number correlation with correlations from literature for various number of row at staggered bundle arrangement

\begin{tabular}{|c|c|c|c|c|c|c|c|c|}
\hline Type & $\mathrm{Z}$ & $\begin{array}{l}\mathrm{Re} \\
\text { number }\end{array}$ & $\begin{array}{l}\text { Nu Eq. } \\
\text { present study }\end{array}$ & $\begin{array}{l}\text { Nu fluent } \\
\text { (Mon, 2003) }\end{array}$ & $\begin{array}{l}\text { Nu equatin } \\
\text { (Mon, 2003) }\end{array}$ & $\begin{array}{l}\text { Nu equation (Briggs } \\
\text { and Young, 1963) }\end{array}$ & $\begin{array}{l}\text { Nu equatin } \\
\text { (Schmidt, 1963) }\end{array}$ & $\begin{array}{l}\text { Nu equatin } \\
\text { (VDI-GVC,1997) }\end{array}$ \\
\hline S6 & 3 & 8600 & 60.620 & 61.02 & 54.370 & 51.490 & 50.29 & 55.500 \\
\hline S6 & 3 & 17000 & 88.480 & 90.14 & 81.800 & 81.900 & 76.99 & 83.540 \\
\hline S6 & 3 & 43000 & 148.090 & 150.46 & 142.810 & 154.090 & 137.51 & 145.790 \\
\hline S4 & 4 & 5000 & 42.043 & 44.82 & 36.300 & 33.230 & 29.13 & 36.900 \\
\hline S13 & 4 & 5000 & 43.560 & 44.18 & 38.750 & 38.170 & 38.56 & 41.280 \\
\hline S14 & 4 & 5000 & 39.570 & 39.24 & 37.280 & 38.170 & 38.56 & 41.280 \\
\hline S1 & 4 & 8600 & 65.250 & 62.81 & 58.600 & 61.160 & 64.21 & 61.210 \\
\hline S2 & 4 & 8600 & 62.310 & 61.90 & 55.290 & 55.220 & 54.12 & 57.160 \\
\hline S3 & 4 & 8600 & 59.850 & 59.60 & 52.910 & 51.630 & 47.56 & 54.280 \\
\hline $\mathrm{S} 4$ & 4 & 8600 & 56.800 & 57.69 & 50.260 & 48.070 & 40.88 & 51.090 \\
\hline S5 & 4 & 8600 & 55.080 & 55.96 & 48.880 & 46.350 & 37.62 & 49.420 \\
\hline S6 & 4 & 8600 & 62.830 & 62.43 & 54.370 & 51.490 & 50.29 & 55.500 \\
\hline S7 & 4 & 8600 & 60.570 & 59.43 & 57.99 & 68.628 & 66.92 & 62.230 \\
\hline S8 & 4 & 8600 & 47.330 & 46.610 & 44.98 & 49.890 & 52.78 & 53.980 \\
\hline S9 & 4 & 8600 & 62.630 & 61.85 & 55.52 & 58.520 & 54.51 & 57.320 \\
\hline $\mathrm{S} 10$ & 4 & 8600 & 62.173 & 62.04 & 55.18 & 54.090 & 53.93 & 57.080 \\
\hline $\mathrm{S} 11$ & 4 & 8600 & 56.270 & 56.76 & 53.17 & 55.220 & 51.89 & 56.200 \\
\hline $\mathrm{S} 12$ & 4 & 8600 & 63.770 & 62.31 & 55.79 & 55.220 & 54.57 & 57.350 \\
\hline S13 & 4 & 8600 & 58.870 & 57.40 & 53.65 & 55.220 & 54.12 & 57.160 \\
\hline $\mathrm{S} 1$ & 4 & 17000 & 95.250 & 93.30 & 88.20 & 97.290 & 98.31 & 92.120 \\
\hline S2 & 4 & 17000 & 90.960 & 91.01 & 83.22 & 87.840 & 82.861 & 86.037 \\
\hline S3 & 4 & 17000 & 87.430 & 86.57 & 79.63 & 82.120 & 72.81 & 81.700 \\
\hline S4 & 4 & 17000 & 82.920 & 81.65 & 75.64 & 76.470 & 62.59 & 76.900 \\
\hline S5 & 4 & 17000 & 80.460 & 78.53 & 73.57 & 116.850 & 57.60 & 74.390 \\
\hline S6 & 4 & 17000 & 91.710 & 92.27 & 81.84 & 81.900 & 76.99 & 83.540 \\
\hline S7 & 4 & 17000 & 88.420 & 88.20 & 87.28 & 109.150 & 102.46 & 93.660 \\
\hline S8 & 4 & 17000 & 81.240 & 80.24 & 83.234 & 94.530 & 96.34 & 98.230 \\
\hline S9 & 4 & 17000 & 91.420 & 90.85 & 83.57 & 93.081 & 83.46 & 86.280 \\
\hline $\mathrm{S} 10$ & 4 & 17000 & 90.750 & 91.21 & 83.05 & 86.040 & 82.56 & 85.910 \\
\hline S11 & 4 & 17000 & 82.140 & 82.64 & 80.03 & 87.840 & 79.44 & 84.600 \\
\hline $\mathrm{S} 12$ & 4 & 17000 & 92.640 & 92.24 & 83.98 & 87.840 & 83.56 & 86.320 \\
\hline $\mathrm{S} 13$ & 4 & 17000 & 85.920 & 86.53 & 80.76 & 87.840 & 82.86 & 86.030 \\
\hline S14 & 4 & 17000 & 78.550 & 76.56 & 77.70 & 87.840 & 82.86 & 86.030 \\
\hline $\mathrm{S} 1$ & 4 & 43000 & 159.180 & 156.65 & 153.92 & 183.030 & 175.59 & 160.760 \\
\hline S2 & 4 & 43000 & 152.240 & 153.33 & 145.23 & 165.250 & 147.99 & 150.140 \\
\hline S3 & 4 & 43000 & 146.330 & 146.17 & 138.97 & 154.500 & 130.05 & 142.570 \\
\hline $\mathrm{S} 4$ & 4 & 43000 & 138.780 & 134.76 & 132.01 & 143.860 & 111.79 & 134.200 \\
\hline S5 & 4 & 43000 & 134.670 & 130.23 & 128.38 & 138.710 & 102.88 & 129.810 \\
\hline S6 & 4 & 43000 & 153.500 & 153.95 & 142.81 & 154.090 & 137.51 & 145.790 \\
\hline S7 & 4 & 43000 & 147.990 & 151.77 & 152.31 & 205.350 & 183.00 & 163.450 \\
\hline S8 & 4 & 43000 & 133.430 & 131.12 & 139.87 & 142.230 & 143.87 & 147.110 \\
\hline S9 & 4 & 43000 & 153.010 & 152.43 & 145.84 & 175.110 & 149.07 & 150.570 \\
\hline $\mathrm{S} 10$ & 4 & 43000 & 151.890 & 153.89 & 144.93 & 161.870 & 147.46 & 149.920 \\
\hline $\mathrm{S} 11$ & 4 & 43000 & 137.470 & 132.65 & 139.66 & 165.250 & 141.89 & 147.630 \\
\hline $\mathrm{S} 12$ & 4 & 43000 & 155.060 & 158.16 & 146.55 & 165.250 & 149.24 & 150.640 \\
\hline $\mathrm{S} 13$ & 4 & 43000 & 143.810 & 149.60 & 140.93 & 165.250 & 147.99 & 150.140 \\
\hline S14 & 4 & 43000 & 131.470 & 135.80 & 135.60 & 165.250 & 147.99 & 150.140 \\
\hline S1 & 4 & 70000 & 208.560 & 202.38 & 206.19 & 206.190 & 255.06 & 238.100 \\
\hline S2 & 4 & 70000 & 191.780 & 189.36 & 186.16 & 215.300 & 176.36 & 191 \\
\hline S3 & 4 & 70000 & 193.950 & 196.69 & 204.04 & 286.170 & 248.16 & 218.960 \\
\hline S6 & 5 & 8600 & 64.311 & 63.18 & 54.37 & 51.490 & 50.29 & 55.500 \\
\hline S6 & 5 & 17000 & 93.872 & 93.39 & 81.84 & 81.900 & 76.99 & 83.540 \\
\hline S6 & 5 & 43000 & 157.110 & 155.43 & 142.81 & 154.000 & 137.51 & 145.790 \\
\hline S6 & 6 & 8600 & 65.220 & 63.6 & 54.37 & 51.490 & 50.28 & 55.500 \\
\hline S6 & 6 & 17000 & 95.200 & 94.13 & 81.84 & 81.900 & 76.99 & 83.540 \\
\hline S6 & 6 & 43000 & 159.350 & 156.79 & 142.81 & 154.090 & 137.51 & 145.700 \\
\hline
\end{tabular}

Table 6: Comparison Eu number correlation with correlations from literature for various number of row at staggered bundle arrangement

\begin{tabular}{|c|c|c|c|c|c|c|c|}
\hline Type & $Z$ & $\mathrm{Re}$ & $\begin{array}{l}\text { Eu equation } \\
\text { present study }\end{array}$ & Eu Fluent (Mon, 2003) & $\begin{array}{l}\text { Eu equation } \\
\text { (Mon, 2003) }\end{array}$ & $\begin{array}{l}\text { Eu equation (Robinson } \\
\text { and Briggs, 1965) }\end{array}$ & $\begin{array}{l}\text { Eu equation } \\
\text { (Haaf, 1988) }\end{array}$ \\
\hline S6 & 3 & 8600 & 1.53 & 1.53 & 1.49 & 1.98 & 1.54 \\
\hline S6 & 3 & 17000 & 1.30 & 1.32 & 1.27 & 1.59 & 1.30 \\
\hline S6 & 3 & 43000 & 1.05 & 1.00 & 1.01 & 1.191 & 1.03 \\
\hline S4 & 4 & 5000 & 2.16 & 2.30 & 2.33 & 2.47 & 2.61 \\
\hline S13 & 4 & 5000 & 1.87 & 1.66 & 1.72 & 2.67 & 2.52 \\
\hline S14 & 4 & 5000 & 1.57 & 1.62 & 1.33 & 2.043 & 2.83 \\
\hline S1 & 4 & 8600 & 1.71 & 1.6 & 1.72 & 2.96 & 1.96 \\
\hline $\mathrm{S} 2$ & 4 & 8600 & 1.82 & 1.79 & 1.83 & 2.64 & 2.06 \\
\hline
\end{tabular}


J. Eng. Applied Sci., 14 (5): 1538-1548, 2019

\begin{tabular}{|c|c|c|c|c|c|c|c|}
\hline Type & $\mathrm{Z}$ & $\operatorname{Re}$ & $\begin{array}{l}\text { Eu equation } \\
\text { present study }\end{array}$ & Eu Fluent (Mon, 2003) & $\begin{array}{l}\text { Eu equation } \\
\text { (Mon, 2003) }\end{array}$ & $\begin{array}{l}\text { Eu equation (Robinson } \\
\text { and Briggs, 1965) }\end{array}$ & $\begin{array}{l}\text { Eu equation } \\
\text { (Haaf, 1988) }\end{array}$ \\
\hline $\mathrm{S3}$ & 4 & 8600 & 1.88 & 1.88 & 1.93 & 2.38 & 2.15 \\
\hline S4 & 4 & 8600 & 1.90 & 1.96 & 2.05 & 2.08 & 2.28 \\
\hline S5 & 4 & 8600 & 1.90 & 2.02 & 2.12 & 1.92 & 2.36 \\
\hline S6 & 4 & 8600 & 1.96 & 2.00 & 1.99 & 2.64 & 2.06 \\
\hline S7 & 4 & 8600 & 1.45 & 1.37 & 1.44 & 2.64 & 2.06 \\
\hline S8 & 4 & 8600 & 4.00 & 3.91 & 4.12 & 4.81 & 4.61 \\
\hline S9 & 4 & 8600 & 1.83 & 1.79 & 1.85 & 2.64 & 2.06 \\
\hline $\mathrm{S} 10$ & 4 & 8600 & 1.82 & 1.78 & 1.83 & 2.64 & 2.06 \\
\hline S11 & 4 & 8600 & 1.57 & 1.46 & 1.59 & 2.19 & 2.23 \\
\hline $\mathrm{S} 12$ & 4 & 8600 & 1.89 & 1.87 & 1.90 & 2.75 & 2.02 \\
\hline $\mathrm{S} 13$ & 4 & 8600 & 1.65 & 1.57 & 1.51 & 2.25 & 2.20 \\
\hline S1 & 4 & 17000 & 1.46 & 1.41 & 1.46 & 2.39 & 1.65 \\
\hline $\mathrm{S} 2$ & 4 & 17000 & 1.56 & 1.557 & 1.56 & 2.13 & 1.73 \\
\hline S3 & 4 & 17000 & 1.60 & 1.60 & 1.64 & 1.92 & 1.81 \\
\hline S4 & 4 & 17000 & 1.63 & 1.62 & 1.74 & 1.67 & 1.92 \\
\hline S5 & 4 & 17000 & 1.62 & 1.68 & 1.80 & 1.54 & 1.99 \\
\hline S6 & 4 & 17000 & 1.68 & 1.70 & 1.69 & 2.13 & 1.73 \\
\hline S7 & 4 & 17000 & 1.24 & 1.26 & 1.22 & 2.13 & 1.73 \\
\hline S8 & 4 & 17000 & 2.98 & 2.88 & 3.00 & 3.23 & 3.41 \\
\hline s9 & 4 & 17000 & 1.57 & 1.55 & 1.57 & 2.13 & 1.73 \\
\hline $\mathrm{S} 10$ & 4 & 17000 & 1.55 & 1.55 & 1.55 & 2.13 & 1.73 \\
\hline S11 & 4 & 17000 & 1.34 & 1.21 & 1.35 & 1.76 & 1.88 \\
\hline $\mathrm{S} 12$ & 4 & 17000 & 1.62 & 1.62 & 1.62 & 2.21 & 1.70 \\
\hline S13 & 4 & 17000 & 1.41 & 1.42 & 1.28 & 1.81 & 1.86 \\
\hline S14 & 4 & 17000 & 1.14 & 1.21 & 0.99 & 1.38 & 2.09 \\
\hline S1 & 4 & 43000 & 1.19 & 1.19 & 1.16 & 1.78 & 1.31 \\
\hline $\mathrm{S} 2$ & 4 & 43000 & 1.26 & 1.25 & 1.24 & 1.59 & 1.37 \\
\hline S3 & 4 & 43000 & 1.29 & 1.26 & 1.31 & 1.43 & 1.44 \\
\hline S4 & 4 & 43000 & 1.31 & 1.27 & 1.39 & 1.25 & 1.52 \\
\hline S5 & 4 & 43000 & 1.31 & 1.28 & 1.44 & 1.15 & 1.58 \\
\hline S6 & 4 & 43000 & 1.35 & 1.27 & 1.35 & 1.59 & 1.37 \\
\hline S7 & 4 & 43000 & 1.00 & 1.07 & 0.97 & 1.59 & 1.37 \\
\hline S8 & 4 & 43000 & 1.80 & 1.85 & 1.81 & 1.98 & 2.00 \\
\hline s9 & 4 & 43000 & 1.26 & 1.29 & 1.26 & 1.59 & 1.37 \\
\hline $\mathrm{S} 10$ & 4 & 43000 & 1.25 & 1.29 & 1.24 & 1.59 & 1.37 \\
\hline S11 & 4 & 43000 & 1.08 & 1.03 & 1.082 & 1.316 & 1.49 \\
\hline $\mathrm{S} 12$ & 4 & 43000 & 1.30 & 1.35 & 1.29 & 1.65 & 1.35 \\
\hline S13 & 4 & 43000 & 1.14 & 1.16 & 1.02 & 1.35 & 1.47 \\
\hline S14 & 4 & 43000 & 0.95 & 0.81 & 0.79 & 1.03 & 1.65 \\
\hline S1 & 4 & 70000 & 1.05 & 1.08 & 1.04 & 1.53 & 1.16 \\
\hline $\mathrm{S} 2$ & 4 & 70000 & 1.16 & 1.08 & 1.16 & 1.22 & 1.27 \\
\hline S3 & 4 & 70000 & 0.89 & 1.04 & 0.87 & 1.36 & 1.21 \\
\hline S6 & 5 & 8600 & 2.38 & 2.44 & 2.49 & 3.30 & 2.57 \\
\hline S6 & 5 & 17000 & 2.041 & 2.088 & 2.11 & 2.66 & 2.17 \\
\hline S6 & 5 & 43000 & 1.64 & 1.553 & 1.69 & 1.98 & 1.72 \\
\hline S6 & 6 & 8600 & 2.79 & 2.89 & 2.99 & 3.96 & 3.09 \\
\hline S6 & 6 & 17000 & 2.39 & 2.46 & 2.54 & 3.19 & 2.60 \\
\hline S6 & 6 & 43000 & 1.93 & 1.82 & 2.03 & 2.38 & 2.06 \\
\hline
\end{tabular}

Table 7: Comparison Nu number correlation with correlations from literature for various number of row at in line bundle arrangement

\begin{tabular}{|c|c|c|c|c|c|c|c|}
\hline Type & $\mathrm{Z}$ & $\operatorname{Re}$ & $\begin{array}{l}\text { Eu equation } \\
\text { present study }\end{array}$ & Eu Fluent (Mon, 2003) & $\begin{array}{l}\text { Eu equation } \\
\text { (Mon, 2003) }\end{array}$ & $\begin{array}{l}\text { Eu equation (Robinson } \\
\text { and Briggs, 1965) }\end{array}$ & $\begin{array}{l}\text { Eu equation } \\
\text { (VDI-GVC, 1997) }\end{array}$ \\
\hline I & 3 & 8600 & 32.29 & 29.3 & 27.86 & 33.5200 & 32.23 \\
\hline I4 & 3 & 17000 & 50.67 & 46.59 & 41.93 & 51.3200 & 48.36 \\
\hline I4 & 3 & 43000 & 93.58 & 86.52 & 73.16 & 91.6500 & 84.38 \\
\hline I1 & 4 & 8600 & 31.21 & 34.40 & 29.54 & 36.0810 & 33.09 \\
\hline I2 & 4 & 8600 & 30.64 & 32.61 & 27.56 & 31.7000 & 31.42 \\
\hline I3 & 4 & 8600 & 29.82 & 30.75 & 25.39 & 27.2500 & 29.58 \\
\hline I4 & 4 & 8600 & 29.36 & 32.234 & 27.86 & 33.5300 & 32.13 \\
\hline I5 & 4 & 8600 & 38.41 & 38.81 & 35.04 & 44.6100 & 36.02 \\
\hline I6 & 4 & 8600 & 36.95 & 37.39 & 30.55 & 34.5910 & 32.53 \\
\hline I7 & 4 & 8600 & 33.41 & 32.63 & 33.00 & 35.1239 & 36.23 \\
\hline I8 & 4 & 8600 & 29.98 & 29.31 & 31.45 & 36.0700 & 33.08 \\
\hline I9 & 4 & 8600 & 34.28 & 34.88 & 31.78 & 36.0800 & 33.09 \\
\hline I1 & 4 & 17000 & 48.97 & 54.09 & 44.45 & 55.2300 & 49.80 \\
\hline I2 & 4 & 17000 & 48.07 & 51.26 & 41.48 & 48.5400 & 47.29 \\
\hline I3 & 4 & 17000 & 46.79 & 48.07 & 38.22 & 41.7200 & 44.52 \\
\hline I4 & 4 & 17000 & 46.06 & 51.25 & 41.93 & 51.32 & 48.36 \\
\hline
\end{tabular}




\begin{tabular}{|c|c|c|c|c|c|c|c|}
\hline Type & Z & $\mathrm{Re}$ & $\begin{array}{l}\text { Eu equation } \\
\text { present study }\end{array}$ & Eu Fluent (Mon, 2003) & $\begin{array}{l}\text { Eu equation } \\
\text { (Mon, 2003) }\end{array}$ & $\begin{array}{l}\text { Eu equation (Robinson } \\
\text { and Briggs, 1965) }\end{array}$ & $\begin{array}{l}\text { Eu equation } \\
\text { (VDI-GVC, 1997) }\end{array}$ \\
\hline$\overline{\mathrm{I} 5}$ & 4 & 17000 & 60.27 & 60.34 & 52.74 & 68.28 & 54.20 \\
\hline I6 & 4 & 17000 & 57.98 & 57.12 & 45.98 & 52.95 & 48.96 \\
\hline I7 & 4 & 17000 & 57.91 & 51.81 & 49.87 & 54.12 & 56.12 \\
\hline I8 & 4 & 17000 & 47.04 & 47.46 & 47.33 & 55.22 & 49.79 \\
\hline I9 & 4 & 17000 & 53.79 & 54.60 & 47.83 & 55.23 & 49.80 \\
\hline I1 & 4 & 43000 & 90.43 & 99.67 & 77.57 & 98.63 & 86.91 \\
\hline I2 & 4 & 43000 & 88.77 & 93.97 & 72.37 & 86.68 & 82.52 \\
\hline $\mathrm{I} 3$ & 4 & 43000 & 86.40 & 86.27 & 66.69 & 74.51 & 77.67 \\
\hline $\mathrm{I} 4$ & 4 & 43000 & 85.07 & 95.18 & 73.17 & 91.66 & 84.39 \\
\hline I5 & 4 & 43000 & 111.31 & 112.67 & 92.03 & 121.95 & 94.59 \\
\hline I6 & 4 & 43000 & 107.08 & 103.11 & 80.24 & 94.56 & 85.44 \\
\hline I7 & 4 & 43000 & 97.12 & 96.10 & 98.00 & 99.99 & 100.12 \\
\hline I8 & 4 & 43000 & 86.88 & 89.71 & 82.59 & 98.62 & 86.88 \\
\hline I9 & 4 & 43000 & 99.35 & 100.48 & 83.46 & 98.63 & 86.90 \\
\hline I4 & 5 & 8600 & 29.36 & 31.04 & 27.87 & 33.53 & 32.14 \\
\hline I4 & 5 & 17000 & 46.06 & 49.38 & 41.941 & 51.33 & 48.37 \\
\hline I4 & 5 & 43000 & 85.07 & 92.79 & 73.18 & 91.66 & 84.39 \\
\hline
\end{tabular}

Table 8: Comparison Eu number correlation with correlations from literature for various number of row at in line bundle arrangement

\begin{tabular}{|c|c|c|c|c|c|c|}
\hline Type & Z & $\mathrm{Re}$ & $\begin{array}{l}\text { Eu equation } \\
\text { present study }\end{array}$ & $\begin{array}{l}\text { Eu Fluent } \\
\text { (Mon, 2003) }\end{array}$ & $\begin{array}{l}\text { Eu equation } \\
\text { (Mon, 2003) }\end{array}$ & $\begin{array}{l}\text { Eu equation } \\
\text { (Haaf, 1988) }\end{array}$ \\
\hline I4 & 3 & 8600 & 0.91 & 0.93 & 1.021 & 0.96 \\
\hline I4 & 3 & 17000 & 0.81 & 0.80 & 0.87 & 0.81 \\
\hline I4 & 3 & 43000 & 0.70 & 0.74 & 0.70 & 0.64 \\
\hline I1 & 4 & 8600 & 0.99 & 1.07 & 1.26 & 1.28 \\
\hline I2 & 4 & 8600 & 1.03 & 1.11 & 1.34 & 1.34 \\
\hline I3 & 4 & 8600 & 1.04 & 1.11 & 1.44 & 1.42 \\
\hline I4 & 4 & 8600 & 1.06 & 1.14 & 1.36 & 1.28 \\
\hline I5 & 4 & 8600 & 0.82 & 0.82 & 1.02 & 1.28 \\
\hline I6 & 4 & 8600 & 0.91 & 0.91 & 1.15 & 1.39 \\
\hline I7 & 4 & 8600 & 1.21 & 1.24 & 1.25 & 1.27 \\
\hline I8 & 4 & 8600 & 0.84 & 0.81 & 1.11 & 1.36 \\
\hline I9 & 4 & 8600 & 1.18 & 1.09 & 1.67 & 1.36 \\
\hline I1 & 4 & 17000 & 0.89 & 0.94 & 1.08 & 1.08 \\
\hline I2 & 4 & 17000 & 0.93 & 0.94 & 1.14 & 1.13 \\
\hline I3 & 4 & 17000 & 0.94 & 0.94 & 1.23 & 1.20 \\
\hline I4 & 4 & 17000 & 0.95 & 0.93 & 1.16 & 1.08 \\
\hline I5 & 4 & 17000 & 0.73 & 0.75 & 0.87 & 1.08 \\
\hline I6 & 4 & 17000 & 0.82 & 0.80 & 0.98 & 1.17 \\
\hline I7 & 4 & 17000 & 0.51 & 0.49 & 0.61 & 0.70 \\
\hline I8 & 4 & 17000 & 0.75 & 0.72 & 0.957 & 1.15 \\
\hline I9 & 4 & 17000 & 1.06 & 0.95 & 1.42 & 1.15 \\
\hline I1 & 4 & 43000 & 0.77 & 0.77 & 0.87 & 0.85 \\
\hline I2 & 4 & 43000 & 0.80 & 0.78 & 0.92 & 0.89 \\
\hline I3 & 4 & 43000 & 0.81 & 0.79 & 0.99 & 0.951 \\
\hline I4 & 4 & 43000 & 0.82 & 0.87 & 0.94 & 0.85 \\
\hline I5 & 4 & 43000 & 0.63 & 0.71 & 0.71 & 0.85 \\
\hline I6 & 4 & 43000 & 0.70 & 0.74 & 0.79 & 0.93 \\
\hline I7 & 4 & 43000 & 0.14 & 0.14 & 0.14 & 0.22 \\
\hline I8 & 4 & 43000 & 0.65 & 0.67 & 0.77 & 0.91 \\
\hline I9 & 4 & 43000 & 0.914 & 0.85 & 1.15 & 0.913 \\
\hline I4 & 5 & 8600 & 1.20 & 1.32 & 1.70 & 1.60 \\
\hline I4 & 5 & 17000 & 1.08 & 1.05 & 1.45 & 1.35 \\
\hline$\underline{\mathrm{I} 4}$ & 5 & 43000 & 0.933 & 0.96 & 1.17 & 1.073 \\
\hline
\end{tabular}

Table 9: Maximum deviation of $\mathrm{Nu}$ number and Eu number correlations, staggered arrangement $\mathrm{Nu}$ correlation

\begin{tabular}{|c|c|c|c|c|c|}
\hline Variables & $\begin{array}{l}\text { Eq. Present } \\
\text { study }(\%)\end{array}$ & $\begin{array}{l}\text { Eq. Mon } \\
(2003)(\%)\end{array}$ & $\begin{array}{l}\text { Eq. Briggs } \\
\text { and Young } \\
(1963)(\%)\end{array}$ & $\begin{array}{l}\text { Eq. } \\
\text { Schmidt } \\
(1963)(\%)\end{array}$ & $\begin{array}{l}\text { Eq. VDI- } \\
\text { GVC(1997) } \\
(\%)\end{array}$ \\
\hline $\begin{array}{l}\text { Maximum } \\
\text { deviation }\end{array}$ & 6 & 19 & 48 & 35 & 17 \\
\hline \multicolumn{6}{|c|}{ Table 10: Staggered arrangement Eu correlation } \\
\hline Variables & $\begin{array}{l}\text { Eq. Presen } \\
\text { study }(\%)\end{array}$ & it Eq. Mon & \multicolumn{2}{|c|}{$\begin{array}{l}\text { Eq. Robinson and } \\
\text { Briggs }(1965)(\%)\end{array}$} & $\begin{array}{l}\text { Eq. Haaf } \\
(1988)(\%)\end{array}$ \\
\hline $\begin{array}{l}\text { Maximum } \\
\text { deviation }\end{array}$ & 18 & 19 & \multicolumn{2}{|l|}{92} & 75 \\
\hline
\end{tabular}

Table 11: In line arrangement Nu correlation

\begin{tabular}{lllll}
\hline Variables & $\begin{array}{l}\text { Eq. Present } \\
\text { study }(\%)\end{array}$ & $\begin{array}{l}\text { Eq. Mon } \\
(2003)(\%)\end{array}$ & $\begin{array}{l}\text { Eq. Schmidt } \\
(1963)(\%)\end{array}$ & $\begin{array}{l}\text { Eq.VDI-GVC } \\
(1997)(\%)\end{array}$ \\
\hline $\begin{array}{l}\text { Maximum } \\
\text { deviation }\end{array}$ & 10 & 22 & 23 & 14 \\
\hline
\end{tabular}

Table 12: In line arrangement Eu correlation

\begin{tabular}{llll} 
Variables & $\begin{array}{l}\text { Eq. Present } \\
\text { study }(\%)\end{array}$ & $\begin{array}{l}\text { Eq. Moon } \\
(2003)(\%)\end{array}$ & $\begin{array}{l}\text { Eq. Haaf } \\
(1988)(\%)\end{array}$ \\
\hline $\begin{array}{l}\text { Maximum } \\
\text { deviation }\end{array}$ & 11 & 53 & 67 \\
\hline
\end{tabular}

\section{CONCLUSION}

A new correlation has been developed to predict the heat transfer for both staggered and in-line arrangements in finned tubes heat exchanger. For both staggered and in-line arrangements, the $\mathrm{Nu}$ number increased with increased Re number at all bundles. But Eu number has an adverse effect. The change in value of Nusselt number or Euler number was very small between the bundles for staggered and in line arrangement. In staggered bundle arrangement S1 when increased fin spacing and decreased fin height provides increased of $\mathrm{Nu}$ number and decreased Eu number. Decreased fin spacing and increased fin height causes increased both $\mathrm{Nu}$ number and $\mathrm{Eu}$ number for I1 bundle in line arrangement. Maximum deviation in Nusselt number and Euler number correlations $(6,18 \%$ for staggered and $10,11 \%$ for in line), respectively.

\section{NOMENCLATURE}

Symbol/Description

$\mathrm{d}=$ Tube diameter $(\mathrm{mm})$

$\mathrm{d}_{\mathrm{f}}=$ Fin diameter $(\mathrm{mm})$

$\mathrm{Eu}=$ Euler number

$\mathrm{h}_{\mathrm{f}}=$ Fin height $(\mathrm{mm})$

$\mathrm{k}=$ Thermal conductivity $\left(\mathrm{W} /\left(\mathrm{m} .{ }^{\circ} \mathrm{K}\right)\right)$ 


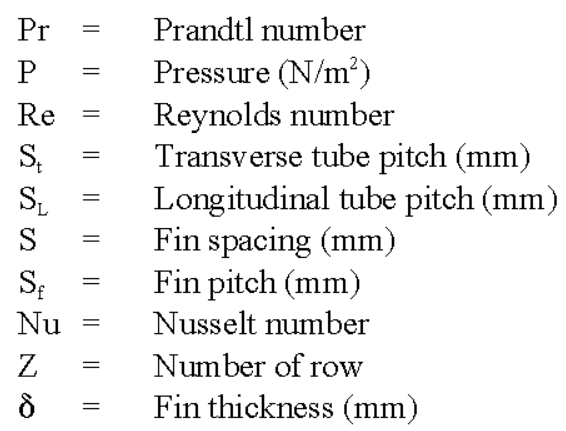

\section{REFERENCES}

Anoop, B., C. Balaji and K. Velusamy, 2015. A characteristic correlation for heat transfer over serrated finned tubes. Anal. Nucl. Energy, 85: 10521065.

Briggs, D.E. and E.H. Young, 1963. Convection heat transfer and pressure drop of air flowing across triangular pitch banks of finned tubes. Chem. Eng. Prog. Symp. Ser., 59: 1-10.

FaJiang, H., C. WeiWu and Y. Ping, 2012. Experimental investigation of heat transfer and flowing resistance for air flow cross over spiral finned tube heat exchanger. Energy Procedia, 17: 741-749.
Haaf, S., 1988. [Heat Transfer in Air Coolers]. In: Handbook of Refrigeration, Plank, R., (Ed.). Springer, Berlin, Germany, ISBN:9783540154778,pp: 435-491 (In German).

Mon, M.S., 2003. Numerical investigation of air-side heat transfer and pressure drop in circular finnedtube heat exchangers. Ph.D Thesis, Freiberg University of Mining and Technology, Freiberg, Germany.

Robinson, K.K. and D.E. Briggs, 1966. Pressure drop of air flowing across triangular pitch banks of finned tubes. Chem. Eng. Prog. Symp. Ser., 64: 177-184.

Schmidt, E., 1963. [The heat transfer to finned tubes and the calculation of tubular heat exchangers (In German)]. Kaltetechnik, 15: 370-378.

Taler, D., 2012. Experimental determination of correlations for mean heat transfer coefficients in plate fin and tube heat exchangers. Arch. Thermodyn., 33: 1-24.

VDI-GVC, 1997. [VDI Heat Atlas: Calculation Sheets for Heat Transfer]. 8th Edn., Springer, Berlin, Germany, ISBN:9783540629009, Pages: 1414 (In German).

Wais, P., 2016. Correlation and numerical study of heat transfer for single row cross-flow heat exchangers with different fin thickness. Procedia Eng., 157: 177184. 